\title{
ALGEBRAS OF FUNCTIONS ON THE UNIT CIRCLE
}

\author{
BY DONALD SARASON
}

1. Introduction. I should like to discuss in this article a few recent developments in function theory on the unit circle. Rather than attempt a broad survey, I have chosen to concentrate on a fairly narrow circle of ideas which I find especially interesting and which seems suitable for presentation to a general audience. This area, while motivated in large part by functional analytic considerations, has a distinctly classical flavor, and some of the questions I shall mention here might have aroused interest forty or fifty years ago if anyone had thought to raise them at the time.

The ideas we shall be concerned with fit into the general contexts of the theory of function algebras and the theory of Hardy spaces and related classes of analytic functions. I have tried to make the bulk of the article intelligible to anyone with a basic knowledge of functional analysis and function theory. Familiarity with a few technical-although by no means esoteric-notions, such as those of a Blaschke product and an inner function, will be helpful to the reader but not indispensable. I have included a few simple proofs here and there where I could do so without being led too far astray. Two minor results below, Theorems 2 and 5, have not to my knowledge been published before.

Notations. We denote the open unit disk by $D$ and its boundary, the unit circle, by $\partial D$. The independent variable on $\partial D$ will be denoted either by $z$ or by $e^{i \theta}$, according to convenience. The basic algebras we shall be concerned with are $C$, the algebra of continuous complex valued functions on $\partial D$, and $L^{\infty}$, the algebra of (classes of) essentially bounded, measurable, complex valued functions with respect to Lebesgue measure on $\partial D$. These are Banach algebras under the supremum and essential supremum norms, respectively. We recall that, by the Gelfand-Naimmark theorem, $L^{\infty}$ is isometrically isomorphic to $C(X)$ for a certain compact Hausdorff space $X$ (the "maximal ideal space" of $L^{\infty}$ ).

We denote by $A$ and $H^{\infty}$ the algebras of functions in $C$ and $L^{\infty}$, respectively, whose Fourier coefficients with negative indices vanish. The functions in $A$ are the boundary functions for the functions that are analytic and uniformly continuous in $D$; those in $H^{\infty}$ are the boundary

An expanded version of an invited address delivered to the 695th meeting of the Society in Seattle, Washington, on June 17, 1972; received by the editors July 14, 1972. Preparation of this article was supported in part by National Science Foundation grant GP-25082.

AMS (MOS) subject classifications (1970). Primary 46J10, 30A76, 30A78. 
functions for the functions that are analytic and bounded in $D$. We shall consistently identify the functions in $A$ and $H^{\infty}$ with their natural analytic extensions into $D$.

2. Wermer's maximality theorem. I should like to begin this account with a theorem discovered approximately twenty years ago by John Wermer [27]. About a year previously, Z. L. Leibenson [17] had proved that if $f$ is any function in $C$ which does not belong to $A$, and if the conjugate function of $f$ is in $C$, then the algebra generated by $f$ and $A$ is dense in $C$. He raised the question whether one can remove the condition that the conjugate function of $f$ be continuous. Wermer answered this in the affirmative, stating his result as follows: If $B$ is a closed subalgebra of $C$ and $A \subset B$, then either $B=A$ or $B=C$.

In general, if $X$ is a compact Hausdorff space, a proper closed subalgebra of $C(X)$ is called maximal if no proper closed subalgebra of $C(X)$ contains it properly. Thus, Wermer's result states that $A$ is a maximal closed subalgebra of $C$. Subsequent to Wermer's discovery, other examples of maximal subalgebras were found, and, in 1960, Hoffman and Singer published a general study of such algebras [16]. (The latter paper contains a bibliography of previous work.) One question considered by Hoffman and Singer is whether, for an arbitrary $X$, every proper closed subalgebra of $C(X)$ is contained in a maximal closed subalgebra. They answered this negatively; the space $X$ in their counterexample is the maximal ideal space of $L^{\infty}$, and the subalgebra is the image of $H^{\infty}$ under the Gelfand transformation of $L^{\infty}$.

Nevertheless, there is a sense in which $H^{\infty}$ is a maximal subalgebra. By adapting a very simple proof they had found of Wermer's theorem, Hoffman and Singer showed that $H^{\infty}$ is maximal among the proper weak-star closed subalgebras of $L^{\infty}$ (where, by the weak-star topology on $L^{\infty}$, we understand, as usual, the weak topology arising from the duality between $L^{\infty}$ and $L^{1}$ ). In fact, they proved the following stronger result (stronger in virtue of the weak-star density of $C$ in $L^{\infty}$ ).

THEOREM 1. If $B$ is a closed subalgebra of $L^{\infty}$ which contains $H^{\infty}$ properly, then $B$ contains $C$.

A short although somewhat indirect argument enables one to deduce Wermer's theorem from the weak-star maximality of $H^{\infty}$. Still, it is natural to seek a more direct link between Theorem 1 and Wermer's theorem by trying to formulate a common generalization which contains both theorems as special cases. An obvious first attempt at such a generalization is to ask whether every closed subalgebra of $L^{\infty}$ which contains $A$ but is not contained in $H^{\infty}$ must contain $C$.

The preceding question, it turns out, has a negative answer. (A counter- 
example will be presented shortly.) Searching a little further, one finds that the Hahn-Banach theorem plays a decisive role.

If $Y$ is a Banach space and $Y_{0}$ is a subspace of $Y$, the Hahn-Banach theorem asserts that any bounded linear functional $\varphi_{0}$ on $Y_{0}$ has a bounded linear extension $\varphi$ to $Y$ satisfying $\|\varphi\|=\left\|\varphi_{0}\right\|$. If there is only one such $\varphi$, then $\varphi_{0}$ is said to have a unique Hahn-Banach extension.

THEOREM 2. Let $B_{0}$ be a subalgebra of $H^{\infty}$ which contains $A$ and which, whenever it contains a function $f$, contains also the function $[f(z)-f(0)] / z$. Let $\varphi_{0}$ be the functional on $B_{0}$ defined by $\varphi_{0}(f)=f(0)$. Let $B$ be a closed subalgebra of $L^{\infty}$ which contains $B_{0}$ but is not contained in $H^{\infty}$. Assume that $\varphi_{0}$ has a unique Hahn-Banach extension to $B$. Then $B$ contains $C$.

We can prove this by borrowing a simple argument G. Lumer [19] has given to establish Wermer's theorem. We reason by contradiction, assuming that $B$ does not contain $C$. Then $B$ does not contain the function $z^{-1}$ because, by the Weierstrass theorem, the uniformly closed algebra generated by $z^{-1}$ and $A$ is $C$. Therefore, the ideal $J$ in $B$ consisting of all functions $z f$ with $f$ in $B$ is proper and so contains no invertible elements of $B$. Hence, each function in $J$ is at a distance of at least 1 from the constant function 1. The Hahn-Banach theorem now implies that there is a bounded linear functional $\varphi$ on $B$ which annihilates $J$ and satisfies $\|\varphi\|=\varphi(1)=1$. Obviously, $\varphi$ is a Hahn-Banach extension of $\varphi_{0}$. But integration with respect to $d \theta / 2 \pi$ defines a Hahn-Banach extension of $\varphi_{0}$ and thus must define the extension $\varphi$. If $f$ is any function in $B$ and $n$ is any positive integer, then the function $z^{n} f$ is in $J$ and so is annihilated by $\varphi$; that is,

$$
\frac{1}{2 \pi} \int_{-\pi}^{\pi} e^{i n \theta} f\left(e^{i \theta}\right) d \theta=0 .
$$

This says that all the Fourier coefficients of $f$ with negative indices vanish, so that $f$ is in $H^{\infty}$. We have arrived at a contradiction to the hypothesis that $B$ is not contained in $H^{\infty}$, and the theorem is proved.

An additional remark will be helpful in interpreting Theorem 2. If $B_{0}$, $\varphi_{0}$ and $B$ are as in Theorem 2 , and if $\varphi$ is any bounded linear extension of $\varphi_{0}$ to $B$, then the condition that $\varphi$ be a Hahn-Banach extension of $\varphi_{0}$ (i.e., that $\|\varphi\|=1$ ) is easily seen to be equivalent to the condition that $\varphi$ be positive, in other words, that $\operatorname{Re} \varphi(f) \geqq 0$ whenever $\operatorname{Re} f \geqq 0$. The following condition is therefore clearly sufficient for $\varphi_{0}$ to have a unique Hahn-Banach extension to $B$ :

$$
\begin{aligned}
\sup \left\{\operatorname{Re} \varphi_{0}(g)\right. & \left.: g \in B_{0}, \operatorname{Re} g \leqq \operatorname{Re} f\right\} \\
& =\inf \left\{\operatorname{Re} \varphi_{0}(g): g \in B_{0}, \operatorname{Re} g \geqq \operatorname{Re} f\right\} \quad \text { for all } f \in B
\end{aligned}
$$


This condition is also necessary, as one can show by an adaptation of the standard proof of the Hahn-Banach theorem. (Condition (1) was first used systematically in the study of function algebras by Lumer [18].)

If $B_{0}=A$ and $B \subset C$, then (1) follows from the uniform density of the real trigonometric polynomials in the real continuous functions on $\partial D$. One thus obtains Wermer's theorem as an immediate corollary of Theorem 2. If $B_{0}=H^{\infty}$ then (1) again holds, so Theorem 1 is also a corollary of Theorem 2 . The validity of (1) in this case, however, is much less immediate; it was first established by Gleason and Whitney [12]. Later, Hoffman [15, p. 182] found an alternative proof that the functional on $H^{\infty}$ of evaluation at 0 has a unique Hahn-Banach extension to $L^{\infty}$. (The latter result was not known at the time [16] was published, preventing the inclusion of Theorem 1 in [16]. Theorem 1 was first published in [15].)

Another case in which (1) holds is when all the functions in $B$ are Riemann integrable. This observation yields the following corollary to Theorem 2: A closed subalgebra of $L^{\infty}$ which contains $A$ and which contains at least one Riemann integrable function not in A contains every function in $C$.

To round out this discussion, I want to mention an example of a closed subalgebra of $L^{\infty}$ which contains $A$, is not contained in $H^{\infty}$, and yet does not contain $C$. The preceding corollary suggests that we might produce such an example by taking the closed subalgebra generated by $A$ and a single function that is not Riemann integrable. Accordingly, we let $K$ be a closed nowhere dense subset of $\partial D$ of positive Lebesgue measure, and we consider the closed subalgebra $B$ of $L^{\infty}$ generated by $A$ and $\chi_{K}$, the characteristic function of $K$. The functions of the form $\chi_{K} g+h$ with $g$ and $h$ in $A$ form a dense subset of $B$. Because $\partial D-K$ is dense in $\partial D$, the distance (in $L^{\infty}$ ) of the function $\chi_{K} g+h$ from the function $z^{-1}$ is at least as large as the distance of $h$ from $z^{-1}$, which is at least 1 . Hence $z^{-1}$ is not in $B$.

Finally, I wish to mention the papers of Cohen [3] and Srinivasan and Wang [26], which contain proofs of Wermer's theorem, each interesting for a different reason. The proof of Srinivasan and Wang makes a connection between Wermer's theorem and the invariant subspace structure of the shift operator. The weak-star maximality of $H^{\infty}$ can also be approached via the invariant subspace structure of the shift operator; see [13, p. 27] and [23].

3. $H^{\infty}+C$. I shall be concerned in the remainder of this article mainly with closed subalgebras of $L^{\infty}$ that contain $H^{\infty}$ properly. By Theorem 1 there is a smallest such algebra, namely, the closed subalgebra of $L^{\infty}$ generated by $H^{\infty}$ and $C$. Somewhat unexpectedly, this algebra turns out to equal $H^{\infty}+C$, the linear hull of $H^{\infty}$ and $C$ (a fact which seems to have 
first been pointed out in [20]). The algebra $H^{\infty}+C$ arises, among other ways, in the study of Toeplitz operators [5], [8] and in a problem in prediction theory investigated by $\mathrm{H}$. Helson and the author [14], [21]. In the present section I shall describe a few of the basic properties of $H^{\infty}+C$.

The key observation needed to prove that $H^{\infty}+C$ is closed in $L^{\infty}$ is this: $\left(^{*}\right)$ If $f$ is any function in $C$, then $\operatorname{dist}(f, A)=\operatorname{dist}\left(f, H^{\infty}\right)$. In other words, the map of $C / A$ into $L^{\infty} / H^{\infty}$ which sends $f+A$ to $f+H^{\infty}$ is an isometry. The range of the preceding map is consequently closed, so $H^{\infty}+C$, the inverse image of that range under the quotient map of $L^{\infty}$ onto $L^{\infty} / H^{\infty}$, must also be closed.

The quotient space $L^{\infty} / H^{\infty}$ can be naturally identified with the bidual of the quotient space $C / A$. Under this identification, the above described map becomes the canonical embedding of $C / A$ into its bidual. Since such canonical embeddings are isometries, the distance equality $(*)$ follows immediately. (See $[\mathbf{1 4}]$ for additional details.) L. Zalcman [28] has pointed out the following more direct proof of (*). For $f$ in $C$ and $h$ in $H^{\infty}$, let $f_{n}$ and $h_{n}$ be the $n$th Cesaro means of the Fourier series of $f$ and $h$. Then

$$
\left\|f-h_{n}\right\|_{\infty} \leqq\left\|f-f_{n}\right\|_{\infty}+\left\|f_{n}-h_{n}\right\|_{\infty} \leqq\left\|f-f_{n}\right\|_{\infty}+\|f-h\|_{\infty},
$$

because the $L^{\infty}$-norms of the Cesaro means of a function do not exceed the $L^{\infty}$-norm of the function. Since $\left\|f-f_{n}\right\|_{\infty} \rightarrow 0$, and since each $h_{n}$ is in $A$, we conclude that $\operatorname{dist}(f, A) \leqq \operatorname{dist}\left(f, H^{\infty}\right)$. The reverse inequality is trivial.

Once one knows that $H^{\infty}+C$ is closed, it is a simple matter to prove that it is an algebra. For $n=1,2, \ldots$, the subspace $z^{-n} H^{\infty}$ is contained in $H^{\infty}+C$. The subspace $\bigcup_{1}^{\infty} z^{-n} H^{\infty}$ is an algebra and its closure, which is also an algebra, is easily seen to contain $H^{\infty}+C$. Since $H^{\infty}+C$ is closed it must equal the closure of $\bigcup_{1}^{\infty} z^{-n} H^{\infty}$, and therefore $H^{\infty}+C$ is an algebra.

In studying a Banach algebra, it is often useful to have a criterion which identifies the invertible elements of the algebra. Such a criterion for $H^{\infty}+C$ has been established and exploited by R. G. Douglas [5]; it involves the behavior inside the unit disk of the harmonic extensions of the functions in $H^{\infty}+C$. We let $P$ denote the Poisson kernel:

$$
P(r, \theta)=\left(1-r^{2}\right) /\left(1-2 r \cos \theta+r^{2}\right) .
$$

For $f$ in $L^{\infty}$, the harmonic extension of $f$ into $D$ is defined by Poisson's formula :

$$
f\left(r e^{i \theta}\right)=\frac{1}{2 \pi} \int_{-\pi}^{\pi} f\left(e^{i t}\right) P(r, \theta-t) d t .
$$


If $f$ is in $H^{\infty}$ this coincides with the analytic extension of $f$ into $D$. We shall henceforth think of the functions in $L^{\infty}$ as extended harmonically into $D$. For $0<r<1$ we define $f_{r}\left(e^{i \theta}\right)=f\left(r e^{i \theta}\right)$.

The key to Douglas' criterion is the observation that the Poisson integral is asymptotically multiplicative on $H^{\infty}+C$. The following theorem expresses the latter property.

THEOREM 3. If $f$ and $g$ are in $H^{\infty}+C$, then $\left\|f_{r} g_{r}-(f g)_{r}\right\|_{\infty} \rightarrow 0$ as $r \rightarrow 1$.

The theorem is an easy consequence of the following lemma.

LEMMA. If $f$ is in $C$ and $g$ is in $L^{\infty}$, then $\left\|f_{r} g_{r}-(f g)_{r}\right\|_{\infty} \rightarrow 0$ as $r \rightarrow 1$.

The lemma can be proved by means of straightforward estimates of Poisson integrals. The proof will not be given here.

The invertibility criterion of Douglas is given by the next theorem.

THEOREM 4. A function $f$ in $H^{\infty}+C$ is invertible in $H^{\infty}+C$ if and only if there is an $r_{0}<1$ such that $f$ is bounded away from 0 in the annulus $r_{0}<|z|<1$.

To establish the necessity of the condition in the theorem, assume that $f$ is invertible in $H^{\infty}+C$, and let $g=f^{-1}$. Then, by Theorem 3, $\left\|f_{r} g_{r}-1\right\|_{\infty} \rightarrow 0$ as $r \rightarrow 1$. If we choose $r_{0}$ so that $\left\|f_{r} g_{r}-1\right\|_{\infty}<\frac{1}{2}$ for $r>r_{0}$, we have $|f(z)|>1 / 2\|g\|_{\infty}$ for $r_{0}<|z|<1$, so $f$ satisfies the condition.

To establish the sufficiency of the condition in the theorem, assume first that $f$ is a function in $H^{\infty}$ which satisfies the condition. Then $1 / f$ is bounded and analytic in an annulus $r_{0}<|z|<1$, so $1 / f$ can be written there as the sum of a function which extends to be bounded and analytic in $D$ and a function which extends to be bounded and analytic in $|z|>r_{0}$. The preceding decomposition yields an expression for $1 / f$ on $\partial D$ as the sum of a function in $H^{\infty}$ and a function in $C$, so $f$ is invertible in $H^{\infty}+C$, as desired.

To complete the proof of Theorem 4, assume that $f$ is any function in $H^{\infty}+C$ satisfying the condition in the theorem, say $|f(z)|>\varepsilon\|f\|_{\infty}$ for $r_{0}<|z|<1$. Because $f$ is in $H^{\infty}+C$ we have $\operatorname{dist}\left(f, z^{-n} H^{\infty}\right) \rightarrow 0$ as $n \rightarrow \infty$, so we can find an $n \geqq 0$ and an $h$ in $H^{\infty}$ such that $\left\|f-z^{-n} h\right\|_{\infty}$ $<\varepsilon\|f\|_{\infty} / 2$. The result proved in the preceding paragraph, together with a straightforward estimate, implies that $h$ is invertible in $H^{\infty}+C$ with $\left\|h^{-1}\right\|_{\infty}<2 / \varepsilon\|f\|_{\infty}$. The last two inequalities combine to give $\left\|z^{n} h f-1\right\|_{\infty}$ $<1$, so $z^{n} h f$ is invertible in $H^{\infty}+C$. Thus $f$ is invertible in $H^{\infty}+C$, as was to be proved.

Among inner functions (i.e., functions in $H^{\infty}$ having unit modulus almost everywhere on $\partial D$ ), the only ones satisfying the condition of Theorem 4 are the finite Blaschke products (see, for example, [25]). Hence, the finite 
Blaschke products are the only inner functions that are invertible in $H^{\infty}+C$. A function in $H^{\infty}$ is invertible in $H^{\infty}+C$ if and only if it is invertible in $L^{\infty}$ and its inner factor is a finite Blaschke product.

Theorem 4 enables one to define an index on $\left(H^{\infty}+C\right)^{-1}$, the group of invertible elements of $H^{\infty}+C$. For $f$ in $C^{-1}$ we define ind $f$, as usual, to be the winding number of $f$ about 0 . For $f$ in $\left(H^{\infty}+C\right)^{-1}$ we define ind $f$ to equal ind $f_{r}$ where $r_{0}<r<1$ and $r_{0}$ is as in Theorem 4. The index on $\left(H^{\infty}+C\right)^{-1}$ has the usual properties of an index, namely, it is stable under small perturbations and it is a homomorphism of $\left(H^{\infty}+C\right)^{-1}$ onto the group of integers.

To the information in Theorem 4 one can add a structural description of the invertible functions in $H^{\infty}+C$ analogous to the known description of the invertible functions in $H^{\infty}$. The invertible functions in $H^{\infty}$ are the outer functions that are invertible in $L^{\infty}$, that is, the functions of the form $\lambda \exp (u+i \tilde{u})$, where $\lambda$ is a nonzero constant, $u$ is a real function in $L^{\infty}$, and $\tilde{u}$ is the conjugate function of $u$. All such functions have index 0 .

If $f$ is an invertible function in $H^{\infty}+C$ then we can write $f=w h$ where $|w|=1$ (a.e.) and $h$ is an invertible function in $H^{\infty}$. Namely, for the function $h$ one takes $\exp \left[\log |f|+i(\log |f|)^{\sim}\right]$; except for a scalar factor of modulus $1, h$ is uniquely determined by $f$. To describe the invertible functions in $H^{\infty}+C$, therefore, we can restrict our attention to unimodular functions.

Any unimodular function in $C$ is of course invertible in $C$ and hence also in $H^{\infty}+C$. A unimodular invertible function in $H^{\infty}+C$ which is discontinuous can be produced as follows. Choose a real function $v$ in $C$ such that $\tilde{v}$ is not continuous. We can write $\exp (i \tilde{v})=\exp (v+i \tilde{v}) \exp (-v)$. In the preceding factorization, the first factor is an invertible function in $H^{\infty}$ and the second factor is an invertible function in $C$. The product, which is discontinuous, is therefore an invertible function in $H^{\infty}+C$. It has index 0 because each of its factors does.

The following theorem gives a complete description of the unimodular invertible functions in $H^{\infty}+C$.

THEOREM 5. Let $w$ be a unimodular invertible function in $H^{\infty}+C$ of index $n$. Then there are real functions $u$ and $v$ in $C$ providing the representation $w=z^{n} \exp [i(u+\tilde{v})]$.

A unimodular function in $H^{\infty}+C$ is invertible if and only if its complex conjugate belongs to $H^{\infty}+C$. Following Douglas, we let $Q C$ (for "quasicontinuous") denote the algebra of functions in $H^{\infty}+C$ whose complex conjugates belong to $H^{\infty}+C$ (that is, the largest $C^{*}$-algebra contained in $H^{\infty}+C$ ). A moment's thought reveals that $Q C$ consists precisely of the bounded functions in $C+\widetilde{C}$. In $C$, any invertible function of index 0 has 
a logarithm, that is, a logarithm which belongs to $C$. The analogous statement for $Q C$ is incorrect, but Theorem 5 says that it is, in a sense, almost correct: Any invertible function in $Q C$ of index 0 has a logarithm in $C+\widetilde{C}$.

To prove Theorem 5 we assume that $\operatorname{dist}\left(w, H^{\infty}\right)<1$. Even if this is not true of $w$, it is true of $z^{k} w$ for $k$ sufficiently large, so the assumption involves no loss of generality. Choose $h$ in $H^{\infty}$ such that $\|w-h\|_{\infty}<1$. Then $\left\|1-h w^{-1}\right\|_{\infty}<1$, so $h w^{-1}$ is invertible in $H^{\infty}+C$ and, in fact, has a logarithm in $H^{\infty}+C$. It follows, in particular, that $h$ is invertible in $H^{\infty}+C$, so the inner factor of $h$ is a finite Blaschke product; that is, $h=b \exp \left(v_{1}+i \tilde{v}_{1}\right)$, where $b$ is a finite Blaschke product and $v_{1}=\log |h|$. Since $h w^{-1}$ has a logarithm in $H^{\infty}+C$ so does $w h^{-1}$, enabling us to write $w h^{-1}=\exp (f+g)$ where $f$ is in $C$ and $g$ is in $H^{\infty}$. Setting $v=v_{1}+\operatorname{Re} g$, we obtain $w=b \exp (f+v+i \tilde{v})$. Since $|w|=1$ we must have $v=-\operatorname{Re} f$, so $v$ is in $C$, and, setting $u_{1}=\operatorname{Im} f$, we have $w=b \exp \left[i\left(u_{1}+\tilde{v}\right)\right]$. Finally, if $n$ denotes the number of zeros that $b$ has in $D$, then $z^{-n} b$ has a logarithm in $C$, say $z^{-n} b=\exp \left(i u_{2}\right)$. Letting $u=u_{1}+u_{2}$, we obtain the desired representation $w=z^{n} \exp [i(u+\tilde{v})]$.

There is a well-known structural description of the unimodular functions in $H^{\infty}$ (i.e., the inner functions) [15], [25]. It is natural to seek an analogous description of the unimodular functions in $H^{\infty}+C$. Theorem 5 can be viewed as a first step in that direction. It prompts the following question, which I have been unable to answer: Can every unimodular function in $H^{\infty}+C$ be factored as the product of an inner function and an invertible function? One cannot expect such a factorization, if it exists, to be unique, because it is possible for the quotient of two distinct inner functions to be an invertible function in $H^{\infty}+C$.

The preceding observation suggests it might be interesting to study the circumstances under which one inner function can divide another one in the algebra $H^{\infty}+C$. Although the situation with regard to divisibility in the algebra $H^{\infty}$ is clear-cut, things are much more complicated in $H^{\infty}+C$. For instance, in $H^{\infty}+C$, the inner function $\exp [(z+1) /(z-1)]$ divides and is divisible by a Blaschke product [20]. I do not know the answer to the following question: Can one singular inner function divide another one in $H^{\infty}+C$ without already dividing it in $H^{\infty}$ ?

There is much more one can say about $H^{\infty}+C$, but I want to go on to other topics. I do want to mention in passing an interesting property which was recognized by Kenneth Hoffman. It characterizes the functions in $H^{\infty}+C$ as the functions in $L^{\infty}$ that belong to $H^{\infty}$ "at each point of $\partial D$." The reader will find a more precise formulation of this property, as well as proofs, in [8] and [22].

The algebra $Q C$ appears to merit additional study. One might hope, 
for example, to obtain a reasonably concrete description of the maximal ideal space of $Q C$. It is interesting that the functions in $Q C$ can be characterized in terms of a smoothness property. The characterization comes out of recent work of Fefferman and Stein [10] concerning functions of bounded mean oscillation. To state it, we define, for $f$ in $L^{1}$ and $I$ a subarc of $\partial D$,

$$
\operatorname{av}_{I} f=|I|^{-1} \int_{I} f d \theta,
$$

where $|I|=\int_{I} d \theta$. In addition, we let

$$
\begin{aligned}
& M_{r}(f)=\sup _{|I| \leqq 2 \pi r}|I|^{-1} \int_{I}\left|f-\operatorname{av}_{I} f\right| d \theta, \quad 0<r \leqq 1, \\
& M_{0}(f)=\lim _{r \rightarrow 0} M_{r}(f) .
\end{aligned}
$$

The quantity $M_{1}(f)$ is called the mean oscillation of $f$. In case $M_{1}(f)<\infty$ one says that $f$ has bounded mean oscillation or that $f$ belongs to BMO. In case $M_{0}(f)=0$ one says that $f$ has vanishing mean oscillation or that $f$ belongs to VMO. The basic result of $[10]$ is the equality $\mathrm{BMO}=L^{\infty}$ $+\left(L^{\infty}\right)^{\sim}$. It is comparatively easy to prove from this that VMO $=C+\widetilde{C}$. We thus have the equality $Q C=L^{\infty} \cap \mathrm{VMO}$.

A unimodular function in $Q C$ has the interesting property that the absolute value of its harmonic extension is continuous in $\bar{D}$. In connection with a problem we shall discuss in the next section, R. G. Douglas has asked whether the last statement has a converse. More precisely, if $w$ is in $L^{\infty}$, if $|w|=1$ (a.e.), and if the absolute value of the harmonic extension of $w$ is continuous in $\bar{D}$, must $w$ belong to $Q C$ ? It seems to the author that one should be able to resolve this question by exploiting the connection between $Q C$ and VMO.

If $w$ has the above properties and if $B$ is the closed subalgebra of $L^{\infty}$ generated by $H^{\infty}$ and $w$, one can show that the Poisson integral is asymptotically multiplicative on $B$, in the sense described in Theorem 3. Conversely, one can show that if there is a closed subalgebra of $L^{\infty}$ containing $H^{\infty}+C$ properly on which the Poisson integral is asymptotically multiplicative, then this algebra contains a function $w$ with the above properties which does not belong to $H^{\infty}+C$. Douglas' question, therefore, is equivalent to the question of whether the Poisson integral can be asymptotically multiplicative on a proper superalgebra of $H^{\infty}+C$.

4. Douglas' problem. In [6], Douglas shows how the study of Toeplitz operators on $\mathrm{H}^{2}$ leads to an investigation of certain closed subalgebras of $L^{\infty}$ which contain $H^{\infty}$. He defines his algebras in terms of their generators, 
and he asks whether every closed subalgebra of $L^{\infty}$ which contains $H^{\infty}$ might not be of the kind he is considering. The question raised by Douglas is still unresolved. In the remainder of this article I shall discuss the question and describe some work related to it.

For $B$ a closed subalgebra of $L^{\infty}$ which contains $H^{\infty}$, we let $B_{I}$ denote the closed subalgebra generated by $H^{\infty}$ and the inverses of the functions in $H^{\infty}$ that are invertible in $B$. Obviously $B_{I} \subset B$; if $B_{I}=B$ we call $B$ a Douglas subalgebra. Douglas' question is whether the equality $B_{I}=B$ always holds.

The algebra $B_{I}$ can be defined, alternatively, as the closed subalgebra generated by $H^{\infty}$ and the complex conjugates of the inner functions that are invertible in $B$. (This is so because, if a function in $H^{\infty}$ is invertible in $L^{\infty}$, then its outer factor is invertible in $H^{\infty}$, so the function itself is invertible in $B$ if and only if its inner factor is.) Douglas' question can thus be reformulated to read as follows: Is every closed subalgebra of $L^{\infty}$ which contains $H^{\infty}$ generated by $H^{\infty}$ and the complex conjugates of inner functions?

The algebra $H^{\infty}+C$ is obviously a Douglas subalgebra; it is generated by $H^{\infty}$ and the complex conjugate of the inner function $z$. The algebra $L^{\infty}$ is also a Douglas subalgebra. This can be established by the following simple argument due to W. Rudin. It is enough to show that $L_{I}^{\infty}$ contains the characteristic function of every Borel subset of $\partial D$. Let $\chi$ be such a characteristic function, and let $u=\frac{1}{2}-\chi$. Then $f_{n}=1+\exp [n(u+i \tilde{u})]$ $(n=1,2, \ldots)$ is in $H^{\infty}$ and is invertible in $L^{\infty}$. Since $f_{n}^{-1} \rightarrow \chi$ in $L^{\infty}$-norm as $n \rightarrow \infty$, the function $\chi$ belongs to $L_{I}^{\infty}$, as was to be proved.

At this point it is natural to raise the following general question, an affirmative answer to which would entail an affirmative answer to Douglas' question: Let $B, B^{\prime}, B^{\prime \prime}$ be commutative Banach algebras such that $B^{\prime} \subset B \subset B^{\prime \prime}$, and such that $B^{\prime \prime}$ is generated by $B^{\prime}$ and the inverses of the functions in $B^{\prime}$ that are invertible in $B^{\prime \prime}$. Is $B$ necessarily generated by $B^{\prime}$ and the inverses of the functions in $B^{\prime}$ that are invertible in $B$ ? I am embarassed to report that I pondered various aspects of Douglas' question for several years without hitting upon this obvious generalization, which was eventually pointed out by P. R. Halmos. It is fortunate for my peace of mind that the generalized question has a negative answer. To obtain a simple counterexample, take $B^{\prime \prime}=C, B=A$, and $B^{\prime}$ equal to the algebra of functions in $A$ whose first derivatives vanish at the origin.

We can gain a different perspective on Douglas' question by considering it from the point of view of the Gelfand theory. Let $X$ and $Y$ denote the maximal ideal spaces of $L^{\infty}$ and $H^{\infty}$, respectively, with their Gelfand topologies. We shall find it convenient to make the usual identification of the maximal ideals in $X$ and $Y$ with the multiplicative linear functionals 
they induce. Although the spaces $X$ and $Y$ cannot be described in concrete terms, a good deal of information about their structures is available. I shall mention a few pertinent facts; more details can be found in Chapter 10 of [15]. Each functional in $Y$ can be thought of as "lying above" a certain point in the closed disk $\bar{D}$; the point in question is the value of thefunctional on the function $z$. Only one functional in $Y$ lies above each point in the open disk $D$, namely, the functional of evaluation at that point. Thus, $D$ may be identified with a subset of $Y$. This identification is, in fact, a homeomorphic embedding of $D$ onto an open subset of $Y$. By the famous corona theorem of Carleson [2], $D$ is dense in $Y$. The space $X$ can also be homeomorphically identified with a subset of $Y$. The identification in question associates with a functional in $X$ its restriction to $H^{\infty}$. Under this identification, $X$ becomes the Shilov boundary of the algebra $H^{\infty}$. By a theorem of Hoffman, each functional $y$ in $Y$ has a unique Hahn-Banach extension to $L^{\infty}$ and so is represented by a unique Borel probability measure $m_{y}$ on $X$. If $\hat{f}$ is the Gelfand transform of the function $f$ in $L^{\infty}$, we extend $\hat{f}$ to $Y$ by defining $\hat{f}(y)=\int \hat{f} d m_{y}$. The extended $\hat{f}$ is continuous.

Let $B$ be a closed subalgebra of $L^{\infty}$ which contains $H^{\infty}$, and let $M(B)$ denote the maximal ideal space of $B$ (or, as we prefer to think of it, the space of multiplicative linear functionals on $B$ ), with the Gelfand topology. The theorem of Hoffman referred to above guarantees that distinct functionals in $M(B)$ have distinct restrictions to $H^{\infty}$. Thus, by associating each functional in $M(B)$ with its restriction to $H^{\infty}$, we can homeomorphically identify $M(B)$ with a subset of $Y$. If $\varphi$ is an inner function, or, for that matter, any unimodular function in $B$, then $\varphi$ is invertible in $B$ if and only if $|\hat{\varphi}|=1$ everywhere on $M(B)$. Conversely, if $B$ is generated by $H^{\infty}$ and the complex conjugates of certain inner functions, then $M(B)$ consists precisely of the set of points in $Y$ at which the Gelfand transforms of the inner functions involved all have unit modulus. Thus, if Douglas' question has an affirmative answer, then $B$ is uniquely determined by $M(B)$, that is to say, the following question has a negative answer: Do there exist distinct closed subalgebras $B$ and $B^{\prime}$ of $L^{\infty}$ which contain $H^{\infty}$ such that $M(B)$ and $M\left(B^{\prime}\right)$ coincide (as subsets of $\left.Y\right)$ ? A theorem of Garnett and Glicksberg [11], although it contains a hypothesis not fulfilled in the present situation, is closely enough related to suggest the possibility that techniques from the general theory of uniform algebras may lead to an answer or at least provide some insight.

The algebra $H^{\infty}+C$ is generated by $H^{\infty}$ and the complex conjugate of the inner function $z$. The Gelfand transform of $z$ has unit modulus except at the points of $D$. Hence, $M\left(H^{\infty}+C\right)=Y-D$. For $B$ a closed subalgebra of $L^{\infty}$ which contains $H^{\infty}$ properly, it is not hard to show that $M(B)=Y-D$ if and only if the Poisson integral is asymptotically 
multiplicative on $B$. The question in the last paragraph, therefore, in the special case where $B^{\prime}=H^{\infty}+C$, reduces to the question raised at the end of the preceding section.

My own intuition has not offered me any strong suggestions concerning Douglas' question. Douglas has told me he suspects the question has a positive answer, but others have the opposite impression. In the absence of an idea for attacking the general question, it is natural to try to answer the question for specific algebras that might be of interest in their own right. I shall describe in the remaining sections of this article what has been done along these lines and some of what has been discovered about the algebras that have been investigated.

5. $H^{\infty}+L_{E}^{\infty}$. For $E$ an arbitrary subset of $\partial D$, let $L_{E}^{\infty}$ denote the set of functions in $L^{\infty}$ that are continuous at each point of $E$. Davie, Gamelin and Garnett prove in [4] that the closed subalgebra of $L^{\infty}$ generated by $H^{\infty}$ and $L_{E}^{\infty}$ coincides with $H^{\infty}+L_{E}^{\infty}$, the linear hull of $H^{\infty}$ and $L_{E}^{\infty}$. In the very special case where $E=\partial D$ one obtains from this, of course, the statement that $H^{\infty}+C$ is a closed subalgebra of $L^{\infty}$. The result quoted above is itself a special case of a more general result from [4] in which the unit disk is replaced by an arbitrary bounded open subset of the complex plane.

Davie, Gamelin and Garnett prove, in addition, that $H^{\infty}+L_{E}^{\infty}$ is a Douglas subalgebra by showing that every unimodular function in $L_{E}^{\infty}$ can be uniformly approximated by functions of the form $b_{1} \bar{b}_{2}$ where $b_{1}$ and $b_{2}$ are Blaschke products whose zeros do not cluster at any point of $E$. The special case of the latter result where $E=\varnothing$ is due to Douglas and Rudin [7]. The more elementary case where $E=\partial D$ can be found, essentially, in [14].

6. $A_{1}$. The real line, rather than the unit circle, provides the natural setting for the next algebra we shall consider. Let $L^{\infty}(\boldsymbol{R})$ denote the Lebesgue space of essentially bounded measurable functions with respect to Lebesgue measure on $\boldsymbol{R}$, and let $H^{\infty}(\boldsymbol{R})$ denote the subspace of boundary functions for bounded analytic functions in the upper half-plane. We define $A_{1}$ to be the closed subalgebra of $L^{\infty}(\boldsymbol{R})$ generated by $H^{\infty}(\boldsymbol{R})$ and the bounded uniformly continuous functions on $\boldsymbol{R}$. By a theorem of Kober $\left[1\right.$, p. 249], $A_{1}$ is the Douglas subalgebra generated by $H^{\infty}(R)$ and the complex conjugate of the inner function $e^{i z}$. (Thus, by applying a Cayley transformation, one can transform $A_{1}$ into the closed subalgebra of $L^{\infty}$ generated by $H^{\infty}$ and the complex conjugate of the inner function $\exp [(z+1) /(z-1)]$.)

The algebra $A_{1}$ arises in connection with the study of certain Toeplitz operators; the connection and a few properties of $A_{1}$ are described in [9]. 
In some respects $A_{1}$ is the analogue for $\boldsymbol{R}$ of the algebra $H^{\infty}+C$. For example, in analogy with Theorem 4 , a function in $A_{1}$ is invertible if and only if its Poisson integral is bounded away from 0 in some strip $0<\operatorname{Im} z<\varepsilon$.

The Blaschke products (for the upper half-plane) that are invertible in $A_{1}$ can be characterized in several ways. If a Blaschke product $b$ is invertible in $A_{1}$ then, by the invertibility criterion just mentioned, the zeros of $b$ must tend to $\infty$. If $b$ is a Blaschke product whose zeros tend to $\infty$, then the following conditions are equivalent :

(i) $b$ is invertible in $A_{1}$;

(ii) $b$ is uniformly continuous on $\boldsymbol{R}$;

(iii) $b^{\prime}$ is bounded on $\boldsymbol{R}$.

The Blaschke products whose complex conjugates together with $H^{\infty}(\boldsymbol{R})$ generate $A_{1}$ can also be characterized in simple terms. If $b$ is a Blaschke product which is invertible in $A_{1}$, then the following conditions are equivalent :

(i) $A_{1}$ is the closed subalgebra of $L^{\infty}(\boldsymbol{R})$ generated by $H^{\infty}(\boldsymbol{R})$ and $\bar{b}$;

(ii) $|b|$ is bounded away from 1 in every half-plane $\operatorname{Im} z>\varepsilon>0$;

(iii) $b^{\prime}$ is bounded away from 0 on $\boldsymbol{R}$.

7. $B_{1}$. We return to the unit circle. Let $C_{1}$ denote the subalgebra of $L^{\infty}$ consisting of the functions that are continuous except possibly at the point $z=1$ and have one-sided limits at that point. Let $B_{1}$ denote the closed subalgebra of $L^{\infty}$ generated by $H^{\infty}$ and $C_{1}$. The algebra $B_{1}$ is studied in my recent paper [24]. I began that study in the hope that $B_{1}$ would fail to be a Douglas subalgebra. I had the feeling that the algebras $H^{\infty}$ and $C_{1}$ do not "belong together" because a function in $H^{\infty}$ cannot have a jump discontinuity. Thus, I reasoned, by putting these two algebras together one could, perhaps, make something go wrong.

My intuition was completely misleading: The algebra $B_{1}$ is a Douglas subalgebra. The paper [24] contains a proof of this and of some related properties of $B_{1}$. For instance, an invertibility criterion for $B_{1}$ similar to the one for $H^{\infty}+C$ given by Theorem 4 is obtained. The inner functions that are invertible in $B_{1}$ are characterized. They are the Blaschke products whose zeros tend nontangentially to the point $z=1$ at an exponential rate. The Blaschke products whose complex conjugates together with $H^{\infty}$ generate $B_{1}$ are also characterized in terms of the distribution of their zeros.

The techniques used in [24] to study $B_{1}$ apply also to the closed subalgebra of $L^{\infty}$ generated by $H^{\infty}$ and the piecewise continuous functions.

No particular Douglas subalgebras except for the few mentioned in this article have been studied in detail. The general Douglas subalgebra has also been studied to an extent; for this I refer to [6]. 
Acknowledgement. I am indebted to Ronald G. Douglas for stimulating discussions on much of the above material.

ADDED IN PROOF August 29, 1972. The question of Douglas mentioned at the end of $\S 3$ has an affirmative answer. The proof is elementary, given the equality $Q C=L^{\infty} \cap \mathrm{VMO}$.

\section{REFERENCES}

1. R. P. Boas, Jr., Entire functions, Academic Press, New York, 1954. MR 16, 914.

2. L. Carleson, Interpolations by bounded analytic functions and the corona problem, Ann. of Math. (2) 76 (1962), 547-559. MR 25 \# 5186.

3. P. J. Cohen, A note on constructive methods in Banach algebras, Proc. Amer. Math. Soc. 12 (1961), 159-163. MR 23 \# A1827.

4. A. M. Davie, T. W. Gamelin and J. Garnett, Distance estimates and pointwise bounded density (to appear).

5. R. G. Douglas, Toeplitz and Wiener-Hopf operators in $H^{\infty}+C$, Bull. Amer. Math. Soc. 74 (1968), 895-899. MR 37 \# 4648.

6. R. G. Douglas, On the spectrum of Toeplitz and Wiener-Hopf operators, Abstract Spaces and Approximation (Proc. Conf., Oberwolfach, 1968), Birkhäuser, Basel, 1969, pp. 53-66. MR 41 \#4274.

7. R. G. Douglas and W. Rudin, Approximation by inner functions, Pacific J. Math. 31 (1969), 313-320. MR 40 \#7814.

8. R. G. Douglas and D. Sarason, Fredholm Toeplitz operators, Proc. Amer. Math. Soc. 26 (1970), 117-120. MR 41 \# 4275.

9. A class of Toeplitz operators, Indiana Univ. Math. J. 20 (1971), 891-895.

10. C. Fefferman and E. M. Stein, (in preparation).

11. J. Garnett and I. Glicksberg, Algebras with the same multiplicative measures, J. Functional Analysis 1 (1967), 331-341. MR 36 \#691.

12. A. M. Gleason and $\mathrm{H}$. Whitney, The extension of linear functionals on $H^{\infty}$, Pacific J. Math. 12 (1962), 163-182. MR 25 \#5408.

13. H. Helson, Lectures on invariant subspaces, Academic Press, New York, 1964. MR 30 \# 1409.

14. H. Helson and D. Sarason, Past and future, Math. Scand. 21 (1967), 5-16. MR 38 \# 5282

15. K. Hoffman, Banach spaces of analytic functions, Prentice-Hall Ser. in Modern

Analysis, Prentice-Hall, Englewood Cliffs, N.J., 1962. MR 24 \# A2844.

16. K. Hoffman and I. M. Singer, Maximal algebras of continuous functions, Acta Math. 103 (1960), 217-241. MR 22 \#8318.

17. Z. L. Leibenzon, On the ring of continuous functions on a circle, Uspehi Mat. Nauk 7 (1952), no. 4 (50), 163-164. (Russian) MR 14, 386.

18. G. Lumer, Analytic functions and Dirichlet problem, Bull. Amer. Math. Soc. 70 (1964), 98-104. MR 28 \# 1509.

19. On Wermer's maximality theorem, Invent. Math. 8 (1969), 236-237. MR 40 \#4769.

20. D. Sarason, Generalized interpolation in $H^{\infty}$, Trans. Amer. Math. Soc. 127 (1967),

179-203. MR 34 \# 8193

21. — An addendum to "Past and future", Math. Scand. 30 (1972), 62-64.

22. - On products of Toeplitz operators, Acta Sci. Math. (Szeged) (to appear).

23. —_ Invariant subspaces (to appear).

24. - Approximation of piecewise continuous functions by quotients of bounded analytic functions, Canad. J. Math. 24 (1972), 642-657.

25. W. Seidel, On the distribution of values of bounded analytic functions, Trans. Amer. Math. Soc. 36 (1934), 201-226.

27. J. Wermer, On algebras of continuous functions, Proc. Amer. Math. Soc. 4 (1953), 866-869. MR 15, 440.

28. L. Zalcman, Bounded analytic functions on domains of infinite connectivity, Trans. Amer. Math. Soc. 144 (1969), 241-269. MR 40 \# 5884.

Department of Mathematics, University of California, Berkeley, California 94720 